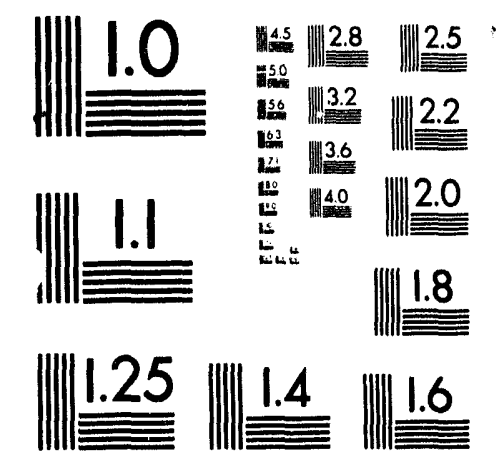



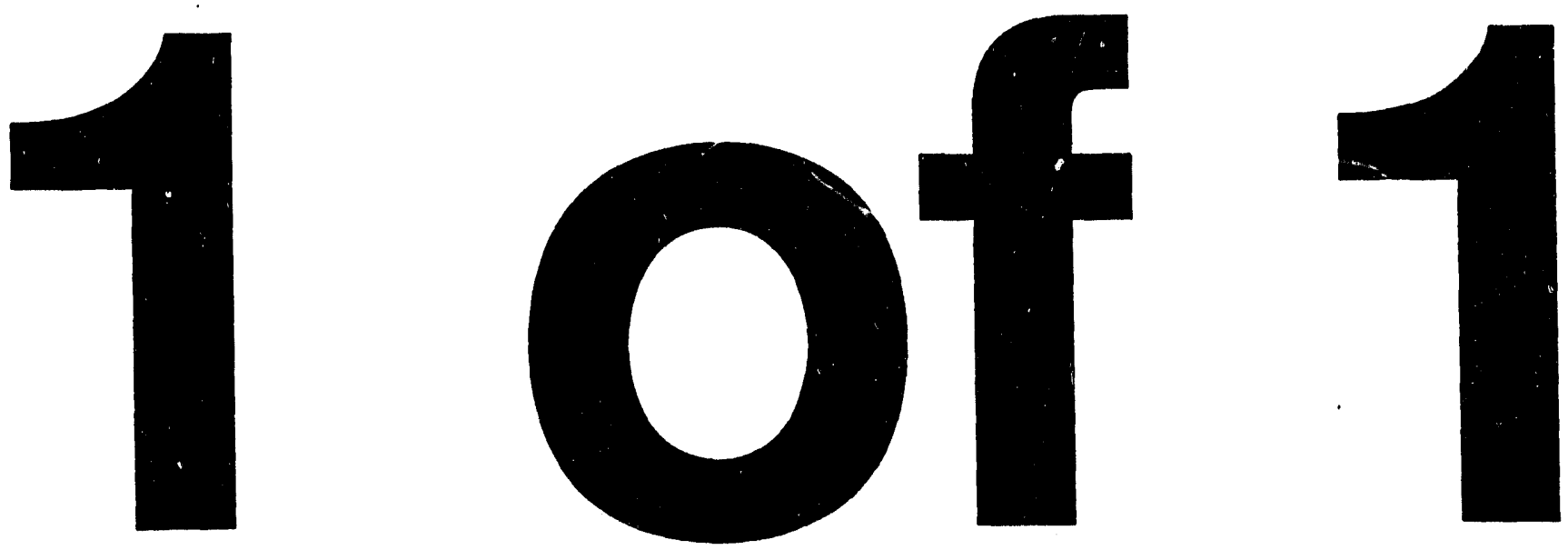
DOE/ER/75787 - T TI

\author{
Department of Nuclear Engineering \\ Massachusetts Institute of Technology \\ Cambridge, MA 02139
}

Tel. $617 / 253-5824$

Fax. 617/258-8863

\title{
PROGRESS REPORT \\ IMPROVING HUMAN RELIABILITY THROUGH \\ BETTER NUCLEAR POWER PLANT \\ SYSTEM DESIGN
}

\author{
Prof. Michael W. Golay
}

10 October 1993

Prepared for

the U.S. Department of Energy

Agreement No. DE-FG02-92ER75787.A001

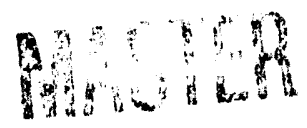

NOTICE: This report was prepared as an account of work sponsored by the United States Government. Neither the United States nor the Department of Energy, nor any of their employees, nor any of their contractors, subcontractors or their employees, makes any warranty, expressed or implied, or assumes any legal liabiity or responsibility for the accuracy, completeness, or usefulness of any information, apparatus, product or process disclosed or represents that its use would not infringe privately-owned rights. 


\title{
PROGRESS REPORT
}

\section{IMPROVING HUMAN RELIABILITY THROUGH BETTER NUCLEAR POWER PLANT SYSTEM DESIGN}

\author{
Principal Investigator: Prof. Michael W. Golay, MIT
}

\begin{abstract}
The project on "Development of a Theory of the Dependence of Luman Reliability upon System Designs as a Means of Improving Nuclear Power Plant Performance" has been undertaken in order to address the important problem of human error in advanced nuclear power plant designs. Most of the creativity in formulating such concepts has focused upon improving the mechanical reliability of safety related plant systems. However, the lack of a mature theory has retarded similar progress in reducing the likely frequencies of human errors. The main design mechanism used address this class of concerns has been to reduce or eliminate the human role in plant operations and accident response.
\end{abstract}

The plan of wort: being pursued in this project is to perform a set of experiments involving human subject who are required to operate, diagnose and respond to changes in computer-simulated systems, relevant to those encountered in nuclear power plants, which are made to differ in complexity in a systematic manner. The computer program used to present the problems to be solved also records the response of the operator as it unfolds. Ultimately this computer is also to be used in formulating the results of the project.

This work is focused upon nuclear power plant applications. However, the persuasiveness of human errors in using all sorts of electro-mechanical machines gives it a much greater potential importance. Because of this we are attempting to pursue our work in a fashion permitting broad generalizations.

\section{INTRODUCTION}

The project is currently proceeding substantially as outlined in the initial project proposal (enclosed in Appendix A). Thus, the discussion of the Progress Report will focus only upon aspects of the work which have deviated from the initial plan of work. In all important aspects the goals, motivations and plan of work remain as initially proposed.

\section{PROGRESS TO-DATE}

The main deviation from the initial plan of work experienced to-date is that the effective start of work was delayed by approximately four months from the intended start date of 1 October 1992. This delay was encountered because it proved to be impossible during the fall 1992 semester to hire a sufficiently skilled graduate Research Assistant to perform the planned work.

The reason was that the funding start date was one month later than the start of the fall semester. At that time the pool of graduate students who had been unable to obtain funding 
on alternative projects from an earlier date, and who were still interested in the substance of the project was too small and of too poorly prepared to justify starting work at that time.

Consequently, the start of work was delayed until the following semester, when an highly qualified graduate student, Eric Hermanson, was recruited into the project. Since February 1993 we have been following the initial plan of work and have been making good progress.

Accomplishments to-date are described below.

We have completed programming the initial version of the computer program for use in testing the ability of human subjects to interact with dynamic systems of differing complexity. The program is written in the highly flexible and user friendly object oriented language NEXTSTEP. Doing this permits us to improve upon the initially proposed approach of using Macintosh-based software for our work., in the sense of obtaining a much more versatile and conformable environment for the human subjects to interact with the systems which they must address in our tests.

These improvements are possible mainly as a result of unexpected changes in the computer industry. As a result much more sophisticated software and hardware is available at reasonable costs than had been anticipated initially. The expected result is an improved research product resuiting from our greater ability to separate test system-dependent influences in the data obtained from influences arising due to limitations of the computer and control system being used for our work.

The system being simulated obeys the general control law of Eq.1, of Appendix A. For our initial set of tests the program has been cast in the particular form of a thermal hydraulic system, involving flows of heat and coolant such as occurs with a steam generator. We are now testing and optimizing the program and to be sure that it is ready for use with the initial class of human subjects. The program behaves well under most conditions, however, for very rapid disturbances it provides unstable simulations. We are currently trying to remove these instabilities before moving on to tests involving our first class of human subjects.

Questions to be investigated include those concerning:

1. The range of system conditions under which successful, or partially successful, control is possible

2. The range of system perturbations and failures for which the human subjects are able to diagnose the new system state and required corrective actions correctly (taking into account also various formats for display of the system state information)

3. the number of independent systems variables requiring simultaneous operator control.

In each class of inquiry we expect of obtain the operator success frequency as a function of system conditions (i.e., complexity). The initial class of human subjects is expected to start working at the end of October 1993, approximately one month behind schedule. For this purpose we have recently begun recruiting the first class of subjects. As long as feasible we shall try to find them among the graduate student population at MIT.

Thus, our progress to date has been largely consistent with the plan of work initially proposed. 
- ne n a

\section{APPRENDIX A}


PROPOSAL

\section{IMPROVING HUMAN RELIABILITY THROUGH BETTER NUCLEAR POWER PLANT SYSTEM DESIGN}

submitted to

U.S. Department of Energy

by

Department of Nuclear Engineering Massachusetts Institute of Technology

Cambridge Massachusetts 02139

Prof. Michael W. Golay: Principal Investigator

13 April 1992 


\title{
-
}

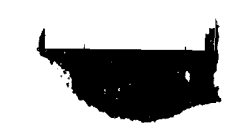

\begin{abstract}
The most important area of needed safety improvements in nuclear power plant technology concerns human errors. The proposed work is concerned with developing quantitative models for the reliability of human operators in diagnosing and controlling systems under upset conditions. It goes beyond the current lore of ergonomics and human factors, by relating human performance to systems design variables. This work is derived from substantial prior research concerned with improving human reliability in nuclear power station operations. The proposed work aims to power plants. The proposed work is based upon a series of experiments involving human subjects attacking system diagnosis and control, as well as maintenance problems. In these experiments the system design and information content of problems confronting operators will be varied and the resulting human performance data correlated into a model for use by system designers. Important applications for upgrades of existing plants as well as design of new plants are foreseen.
\end{abstract}

\section{INTRODUCTION}

The most serious problem blocking improvement of nuclear power technology is that of understand how to ensure adequate human reliability. For a researcher this is a particularly frustrating topic because its importance rarely translates into the justification for funding to attack the problem. This is because our research funding system will provide support to solve an easy problem, but not to make progress on a hard one. Hopefully the response to this proposal will disprove that rule.

Low human reliability is a well-recognized problem in the operation of nuclear power plants. Human reliability failures played major roles in both the Three Mile Island (TMI) and Chernobyl nuclear power plant accidents. Low human reliability was singled-out by the Kemeny Commission report, concerning the TMI accident as a high-priority problem for future research. In unpublished reports by the Institute for Nuclear Power Operations human errors have been identified as being associated with approximately half of the operational unavailability of the existing U.S. nuclear power plants, contributing equally through actions in maintenance and operations.

Human errors in operations, construction, maintenance and fabrication have also been identified as major concerns for future passively safe nuclear power stations. Several such power reactor concepts are under current development. They offer great promise for improvements in the mechanical reliability of systems intended to perform essential safety functions (i.e., power maneuvering, reactor shutdown, post-shutdown reactor cooling, post-shutdown containment building cooling). However, they do not offer promise of corresponding improvements in the human influences upon safety-related reliability.

Thus, low human reliability has been a serious problem with the current generation of nuclear power plants. Concerns that this will continue to be the case cloud the promise of advanced nuclear power stations which are proposed for future deployment. One of the great difficulties posed by such errors is that they can be very hard to anticipate, as they can arise in amazing forms. For example, in the TMI accident the plant operators intentionally disabled the emergency core cooling system as it was operating.

The performance of people is often modeled as if it were a random variable, independent of the systems with which they are dealing. We all know that it is not. Rather, it is clear that some systems are more "user friendly" than others, however, a theory to explain why still remains largely lacking. Through prior work at MIT, upon which this proposal is based, we have made considerable progress in this area. This experience provides the confidence that further valuable contributions can be made. 
The work proposed here will be concerned with creation of the models of the designdependence of human reliability, which can be used as the basis for achieving improvements in nuclear power plant operations and maintenance. Such models are needed by nuclear power plant designers in order to show how different design concepts will affect the expected frequencies of human errors.

Currently the human factors literature, while primarily qualitative, is helpful to designers in identifying classes of design approaches which will be helpful in increasing human reliability. However, the relatively few quantitative models for human reliability which it provides are highly simplified and limited, and can be improved substantially.

This situation is reflected in the current state of probabilistic safety assessment (PSA) modeling, where the treatment of human reliability is an area of major weakness. PSA modeling practices have not yet become standardized; however, the typical PSA will only attempt to treat actions of commission in response to emergencies. Typically actions in operations and maintenance are ignored, as are cognitive and gratuitous errors of all sorts. When these latter classes of errors are taken into account it usually is done by inclusion in a broadly defined top level event within an accident event sequence. In all cases the treatment used is highly subjective, relying substantially upon "expert judgement" for defining which actions to address and for quantifying their expected reliabilities. follows:

The basis of the proposed work is presented in three papers, enclosed in Appendices A-C as

- Appendix A - P.H. Seo.rg, V.P. Manno and M.W. Golay, "Application of a Power Plant Simplification Methodology: The Example of the Condensate Feedwater System," Nuclear Engineering \& Design 110 (1988): 33-46.

- Appendix B - M.W. Golay, P.H. Seong and V.P. Manno, "A Measure of the Difficulty of System Diagnosis and its Relationship to Complexity," Int'l. J. Gen. Systems 16 (1990): 1-23.

- Appendix C - W.G. He and M.W. Golay, "The Dependence of Human Deductive Capabilities Upon Problem Uncertainty," presented at the Meeting on Probabilistic Safety Assessment and Management (PSAM), Beverly Hills, CA (Feb., 1991).

These papers describe work concerned with:

- Improving human operational reliability through better systems design; and

- Understanding fundamental relationships between system complexity and important aspects of human performance.

In examining ways by which plant performance can be impaired it is seen that human contributions to poor performance arise in several ways: in design, construction and fabrication, maintenance and operations. The work of this proposal will be concerned primarily with increasing the reliability of human actions in plant operations and maintenance. This will be done in recognition of the importance of such activities. Particularly, human reliability can potentially be improved more easily in the other areas of nuclear power plant performance than in operations by using available informatic technologies (e.g., those for automated reasoning, or expert systems). Informatic technologies can also be helpful in improving nuclear power plant operations, but by themselves will be insufficient to provide the needed improvements in human reliability. 
One of the greatest impediments to use of a comprehensive approach to nuclear power plant design refinement is that many of the models needed for improvement of human reliability either do not exist or are available in such primitive forms that they are not very useful (e.g., Worledge, 1985). Remedying that defect provides the motivation for the proposed work.

The paper of Appendix A argues that more attractive designs can be created through satisfaction of a more complete set of performance goals, and illustrates how this can be done in the example of the PWR condensate/feedwater system. It shows that this system can be made less complex as an automatic outcome of use of a more complete design refinement approach. In doing this resulting systems can be made more economical and safer. They can also be made easier to operate and to troubleshoot, when they fail to perform as expected. Much of the improved performance of simplified system designs is obtained as a result of increased human reliability, which is obtained as a result of making the system easier to troubleshoot when the system does not perform as required. This paper also shows how the informational entropy of the system can be used in quantification of the expected difficulty of diagnosis of a system which has become upset.

The paper of Appendix B argues that increased human reliability can result when the systems with which human operators interact are made, simpler, or easier to diagnose. It is shown that a measure of the difficulty of diagnosis is the informational entropy, which is defined in terms of the number of states which a system may take and the probabilities of the different states. The expected number of interrogations needed for diagnosis of the condition of an upset system increases exponentially with the entropy. It is also shown that the informational entropy can be used as an index of a version of system complexity.

The paper of Appendix $\mathrm{C}$ uses human subjects to investigate the dependence upon problem complexity of the fraction of attacks upon a deductive problem where success is attained. It is found that the percentage of successful attacks decreases monotonically as the problem complexity (indicated by the problem's initial information) increases. The rate of decrease is approximately independent of the problem structure. However, it is also found that this decreasing trend is observed only at initial information levels exceeding a threshold value. The value of this threshold depends upon the problem structure and the type of data obtained after each iteration in the process of forming a problem solution. The implication for improved system design is to structure systems such that when they must be diagnosed for failure the resulting problem complexity is held below the performance threshold value relevant to that system. It is observed when this is done that nearly perfect problem solutions can be obtained with high reliability. The particular relevant value of the system initial information threshold must be determined through system-specific measurements.

\section{PROPOSED WORK}

\section{Background and Overview}

From the work reported in the papers enclosed with this proposal it is seen that substantially improved models of human reliability are needed by nuclear power plant designers. This is true concerning both operations and maintenance in future nuclear power plants. Some directions for their creation are also indicated in these papers. Building upon the results of the papers of Appendices $\mathrm{B}$ and $\mathrm{C}$ we wish initially to investigate the dependence of human reliability in system control upon system complexity, as indicated by the informational entropy. We have established that the difficulty of diagnosis of a failed system grows exponentially with the informational entropy, and that the rates of success in problem solving decrease as problem complexity increases. We suspect that the expected frequency of errors committed by an operator maneuvering a system through a succession of states (e.g., in response to an accident) also 
depends upon the system informational entropy. This dependence is suspected to be especially strong when the set of procerures being executed is only used infrequently, as in nuclear power plant transient and accident situations.

In the proposed work this dependence, and others, will be investigated. This will be done using human subjects interacting with dynamic systems. The experiments to be performed will be designed with the ability to alter both the system and its external environment. The purpose of these experiments is to determine the variation of operator reliability as the nature of the system being addressed is changed. In addition to varying both the number and probabilities of the different system states factors which are expected to be important in affecting human reliability are the following:

- The dynamic behavior of tr ; system;

- The nature, quantity and reliability of information provided to system operators; and

- The relative certitude which an operator is able to associate with the desirability of the various alternative states into which the system might be placed through future maneuvers.

All of these factors will be varied in the experiments of the proposed work. We expect to use microcomputers to both control and record results from the experiments. The proposed initial experiments are described subsequently. Later, more experiments may be designed, depending upon how the initial work progresses. Generally, the experiments of the proposed work are expected to be somewhat similar to those used previously in the Reactor Innovation Project, and reported in Appendices B and C. Such experiments have included diagnosis of failures of the PWR condensate/feedwater system. The initial phase of the proposed work will be concerned with design and performance of the experiments described subsequently. Depending upon the results obtained in the initial series of experiments, additional means of improving human reliability will also be investigated quantitatively through experiments relevant to nuclear power plant operations and maintenance.

\section{Model of System Control by Human Operators}

The pian of the proposed work is focussed upon determination of the degree of validity of the model for the cognitive human control of a system as outlined in Table 1 . Such validation will be sought through attempting to develop system-dependent quantitative models for each action in the overall sequence of control actions. To the extent that it may be found that such models cannot be developed it will be necessary to revise the overall model.

Within the quantitative model for each action system-dependent quantities are expected to include such elements as the frequency of success and efficiency of performing the action. Extensive use of human subjects is anticipated in formulation of these models. A problem such as is described here can have many important independent variables. Among the more important to be addressed in the proposed work are the following:

- System complexity,

- Uncertainty, which can arising from such sources as unreliable signals and incomplete knowledge,

- Optimal deductive strategies and data requirements,

- Use of informatic systems as deductive, performance monitoring and memory aids for human operators, and

- Use of system behavior feedback through system signals of differing number and quality. 
Many additional factors could also be important in affecting the reliability of human performance. Among those often studied under the disciplinary umbrella of human factors, which will not be addressed in the proposed work are the following: danger, urgency, comfort, stress, distractions, man-machine interface factors, and education and training levels.

\section{Initial Experiments of the Proposed Work}

The proposed work will emphasize use of human subjects (operators) to investigate the influences of such independent factors as those listed previously. These experiments will concentrate on understanding better how people control a system under a broad range of conditions. The essential actions of control are outlined in Table 1. In the planned experiments both the nature of the system being controlled and its initial and desired states will be varied. Data will then be obtained indicating how successfully and efficiently the experimental subjects perform the actions listed in Table 1.

All experiments are expected to be performed using personal computers to present the human subjects with system control problems to which to respond. The resulting system behavior will be calculated in real-time using the computer, and taking into account control actions made by the operator (communicated via keyboard and mouse). Through this process a record of both the problem presented and the operators' responses can be obtained automatically for subsequent analysis. This method has been used very effectively in the previous work reported in Appendices $A$ to $C$. The first stage of work will he design of the experiment, and construction of computer programs for both system behavior simulation and communication with the operator and acquisition of his performance data.

The tests to be performed in these experiments are concerned with diagnosis and control of a general system. However, many tasks in nuclear power station operations and maintenance have important aspects which correspond closely to elements of the problems to be investigated in this work. The work is structured in a general way in order to increase the utility of its results, and to permit very careful testing of the conclusions which are expected to be obtained.

The system to be used for this work (at least initially) is that depicted in Figure 1. It is a general non-linear amplifying system, using feedback which may be either positive or negative, depending upon the experimental case to be investigated. The variables of this system are the following:

- Time, $t$

- The input signal (controlled externally), $V_{\mathrm{i}}(t)$

- The source signal (controlled externally), $S(t)$

- The output signal, $V(t)$

- The gain of the amplifier, $G\left(V\left(t^{\prime}\right)\right)$

This system obeys the relationship:

$$
d V(t) / d t=V_{\mathrm{i}}(t)^{*} G\left(V\left(t^{\prime}\right)\right)+S(t)
$$

subject to the initial conditions

$$
V_{\mathrm{i}}(o)=V_{\mathrm{i}-\mathrm{o}}, S(o)=S_{\mathrm{O}} .
$$


Table 1

\section{MODEL FOR PROCESS OF COGNITIVE HUMAN CONTROL OF A SYSTEM*}

Action 1: Perception of system status indicator signals and signals from the external environment relevant to the system, and testing that these perceptions are correct

Action 2: Use of a system status model, dependent upon the signals perceived in Action 1, to deduce the system condition (with associated uncertainty)

Action 3: Use of a model for determination of the desired system status, depending upon the results of Actions 1 and 2, to determine whether:
A) The system status is desirable (next, perform any actions required to maintain the status; then, return to action 1)
B) The system status is undesirable (next, proceed to Action 4)

Action 4: Use of a system behavior model to identify the most desired sequence of actions sufficient to maneuver the system to the desired status, dependent upon the results of Action 3

Action 5: Implementation of the desired sequence of control maneuvers actions (first, implement the next control maneuver; then, return to Action 1)

\footnotetext{
* The control process consists of continual repetition of the sequence of cognitive Actions 1-5. The informational results of each action of this sequence are typically accompanied by an dissociated level of uncertainty, which can propagate throughout subsequent actions.
} 


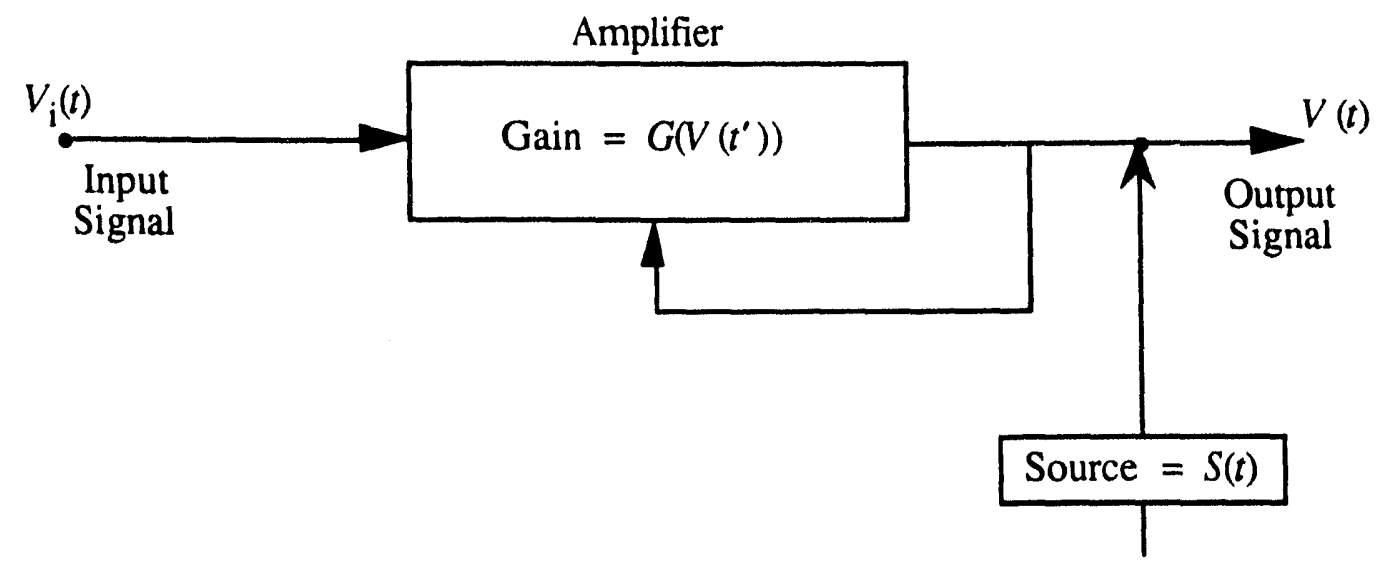

System Control Law:

$$
\begin{aligned}
& \frac{d V(t)}{d t}=G\left(V\left(t^{\prime}\right)\right) * V_{\mathrm{i}}(t)+S(t) \\
& G\left(V\left(t^{\prime}\right)\right)=\sum_{\mathrm{j}=0}^{\mathrm{n}} C_{\mathrm{j}} V^{\mathrm{j}}\left(t^{\prime}\right), \\
& V_{\mathrm{i}}(t), S(t)=\text { Known functions, subject to control, } \\
& t^{\prime}=f(t) .
\end{aligned}
$$

Figure 1: Schematic diagram of system to be diagnosed and controlled in the experiments of the proposed work. 
With this system the output signal, $V(t)$, varies in response to changes in $V_{\mathrm{i}}(t)$ and in $G(V(t))$, both of which will be able to varied in real-time by the system operator in the planned experiments.

Different experimental cases will be specified by the choices of the values of $V_{\mathrm{i}-\mathrm{o}}$ and of the coefficients in the equation governing $G(V(t))$. The form of this latter relationship will initially be the following:

$$
G\left(V\left(t^{\prime}\right)\right)=C_{0}+C_{1} * V\left(t^{\prime}\right)+C_{2} * V_{2}\left(t^{\prime}\right)+\ldots . .+C_{\mathrm{n}} * V_{\mathrm{n}}\left(t^{\prime}\right)
$$

where

$$
V\left(t^{\prime}\right)=V(f(t))
$$

and where

$$
t^{\prime}=f(t)
$$

is an arbitrary function.

The inhomogenous term of Eq. $1, S(t)$ represents system sources and sinks of $V$. This term has direct relevance to nuclear power neutronic and thermal control problems. In initial work we shall not use this term. However, in subsequent experiments the relationship

$$
S=\text { constant }
$$

will be investigated. Also, permitting $S(t)$ to be varied by the human operator will be explored. Doubtless, other important forms of Eq. 6 will become evident as our work proceeds and they will be investigated as their value becomes evident.

In the initial work the form of Eq. 5 will be $t^{\prime}=t$. In subsequent work the relationship: $t^{\prime}=$ $t-A$, (where $A=$ constant) will be investigated (as a means of addressing the effects of system inertia). Later other relationships will be used, depending upon the lessons learned in prior efforts.

The system governed by Eq. 1 is chosen for use in the proposed work because it is very general, and can be made typical of many systems in practical use, including those of nuclear reactors. In addition its behavior can be easily simulated numerically, using temporal finitedifference methods. The functional relationship of Eq. 2 is selected for use in the proposed work because it is also very general, having the form of a truncated Taylor series - which can be used as an approximation for an arbitrary function governing $G(V(t))$.

In the planned experiments the system operator will be permitted to vary the values of the coefficients, $C_{0}, \ldots ., C_{\mathrm{n}}$, and of $V_{\mathrm{i}}(t)$ and to query aspects of the altered system condition in realtime by means of manipulating the computer's control devices. At least, in the initial work the operator will be required to attempt to guide the system so that $V(t)$ will lie within a specified range of values after a specified initial time interval following the start of a test. A particular test will consist of (1) presenting the operator with a system governed by a perturbed set of coefficients, $C_{0}, \ldots ., C_{\mathrm{n}}$ and initial value of $V_{\mathrm{j}}(t),(2)$ simulation of the system behavior under the operator's control, and (3) recording of data characterizing the resulting system behavior. These data will subsequently be analyzed in order to determine the average frequency of successful operator responses, and to reflect means of changing this frequency. 
Initially in the proposed work the system will be perturbed only in its initial conditions and coefficients, $C_{0}, \ldots ., C_{\mathrm{n}}$. However, it is expected subsequently to examine the effects of changes introduced during the course of a test. The latter tests will examine the effects of evolution of the system through successive states, and of multiple successive perturbations. In order to maneuver the system from one state to another it will be necessary for the operator to bring the system level, $V$, to within a specified band of values for a specified duration of time. Following a change of system state a set of indicators will be used to provide the operator with information from which he can diagnose the true state of the system. From his prior training he should then be able to determine the correct system control scheme.

For example, using the different systems descrihed above one can have the operator controlling both the reactor power and cooling system coolant flow. Following a reactor trip, at some point the power will decrease to a level such that natural convection would be sufficient for reactor cooling. At that point a mode shift in the system behavior could be permitted to occur in a test, with the operator being required to respond to the changed conditions.

\section{Classes of Tests}

Three classes of tests are envisioned, each intended to provide data for modeling a different influence upon human reliability:

1. Tests in which the system behavior is known by the operator, but where the system complexity will vary (as in routine operations for plants of differing designs)

2. Tests where the system behavior will be uncertain within a known range, but where the system structure will be kept fixed (as in emergency response operations)

3. Tests where the system state must be diagnosed and maneuvered through a sequence of discrete modes (as in maintenance and some emergency operations)

The product of each set of tests will be a correlation of the data obtained, showing the dependence of the operator's success upon the factors varied. To the extent permitted by resources the effects of simultaneous variation of system complexity and uncertainty will be undertaken, also.

Variation of System Complexity: In the first class of tests the values of the system amplification coefficients, $C_{0}, \ldots, C_{\mathrm{n}}$ will be known by the system operator, and he will have the opportunity to become familiar with the system's performance. In a test the values of certain coefficients will be perturbed initially by a specified amount, and the resulting operator response will be observed. This case corresponds to tha vhere a system is suddenly changed by a wellunderstood perturbation, and the outstanding question concerns how well the operator controls the system. By varying the system conditions we expect to learn more about how systemdependent and external environmental factors affect the ability to control the system. A hypothesis to be tested in this work is that which expects the operator's system control success frequency to become impaired as the number of variables affecting and describing the system performance increases.

Cases to be investigated include the following:

- $C_{0}>0, C_{\mathrm{n}}=0$ for $\mathrm{n}>0$, as in control of a nuclear reactor (with a weak delayed neutron effect)

- $S>0, C_{0}=0, C_{1}<0, C_{\mathrm{n}}=0$ for $\mathrm{n}>1$, as in control of a fluid coolant loop during startup under forced convection conditions

- $S>0, C_{0}<0, C_{1}<0, C_{\mathrm{n}}=0$ for $\mathrm{n}>1$, as in control of a fluid coolant loop during startup under natural convection conditions. 
Variation of System Uncertainty: In the second class of test the values of $V(0)$ and of the coefficients, $C_{\mathrm{o}}, \ldots ., C_{\mathrm{n}}$ will be perturbed from their reference values and the indicated value of $V(t)$ may be in error by amounts which will not be revealed to the operator. Rather, the operator will be told in advance of the range within which each coefficient would be perturbed and the relative range within which $V(t)$ may be in error. However, the operator will be required to rely upon feedback from the system performance and system diagnostic interrogations to indicate the system condition.

This case corresponds to that of a system which is behaving unusually, but where both the cause of the erratic behavior must be determined and simultaneously the system must be controlled stably. Such situations can arise in many versions in nuclear power plants within the contexts of both routine operations and safety-related incidents. In such situation the role of uncertainty is expected to be important in affecting the degree to which successful cc 'tol will be possible.

Maneuvering the System through a sequence of Discrete States: In the first tests of the proposed work the system will be perturbed only in its initial conditions and coefficients, $C_{0}, \ldots$. , $C_{\mathrm{n}}$. However, it is expected subsequently to examine the effects of changes introduced during the course of a test. The latter tests will examine the effects of evolution of the system through successive states, and of multiple successive perturbations. In order to maneuver the system from one state to another it will be necessary for the operator to bring the system level, $V$, to within a specified band of values for a specified duration of time. Following a change of system state a set of indicators will be used to provide the operator with information from which he can diagnose the true state of the system. From his prior training he should then be able to determine the correct system control scheme.

For example, using the different systems described above one can have the operator controlling both the reactor power and cooling system coolant flow. Following a reactor trip, at some point the power will decrease to a level such that natural convection would be sufficient for reactor cooling. At that point a mode shift in the system behavior could be permitted to occur in a test, with the operator being required to respond to the changed conditions.

In such tests the interesting factors to be varied include the difficulty of affecting a mode change, the complexity of the system feedback, and the uncertainty of the knowledge of the system information. The range of variation of independent parameters in the planned tests will be determined during the process of test design and execution, refracting experience gained during performance of the prior tests of classes 1 and 2 .

These problems have direct relevance to the performance of nuclear power stations. From these classes of tests, over a broad range of system conditions, we expect to learn more about how an operator diagnoses a failed system, and about how the uncertainty of the system condition affects the ability to control it successfully. An important hypothesis to be tested by this class of problems expects the rates of success in both system diagnosis and control to suffer significantly as the levels of system complexity and uncertainty increase.

\section{Conclusion}

The work proposed here has a general, fundamental structure. This is done in order that the results obtained may be as general and easily understood and tested as possible. However, it is important to note that the proposed work, and the prior work upon which it is based, is motivated primarily by the problem of rendering future designs of nuclear power plants more resistant to human errors. 
The scope of the proposed work is ambitious but not encyclopedic. Rathei, its limitations are indicative of the difficulty of the problem of improving human reliability. However, given the importance of the problem, better solutions to it must be found. It is important that this be done in a fundamental fashion, and providing quantitative results. The value of the human factors literature has been limited by the fact that much of it is qualitative lore. However, if making such improvements were easy, better answers than are now available would have heen found by this time. The nature of this problem is long-term and in need of vigorous attack. The importance of the problem ensures that it will remain on the research agenda until significant improvements are obtained. Through the proposed work we hope to provide important examples of how this can be done. We have made surprisingly good progress in efforts to-date. However, given the difficulty of this research area, sustained funding is needed if truly valuable research contributions are to be obtained. The DOE research program to which this proposal is submitted offers the possibility of such support. This proposal presents an opportunity, which should be seized, to create a major research contribution.

\section{SCHEDULE AND BUDGET}

The proposed work is expected to require three years for completion, as described in Table 2. The proposed budget is $\$ 429,557$, as summarized in Table 3 . The Principal Investigator for this work is Prof. Michael W. 'uolay. His resume is enclosed in Appendix D.

\section{REFERENCE}

D. H. Worledge, "Some Useful Characteristics of Performance Models," IEEE Conf. on Human Reliability, 1985. 
Table 2

\section{Schedule of Proposed Work}

Time After

Start of Project

Start

6 months

12 months

18 months

24 months

30 months

33 months

36 months

\section{Phase of Work}

Design of Experiments

Start of Class 1 Experiments

First Annual Report; Start of Class 2 Experiments

Start of Class 3 Experiments

Second Annual Report; End of Class 1 Experiments

End of Class 2 Experiments

End of Class 3 Experiments

Final Report 

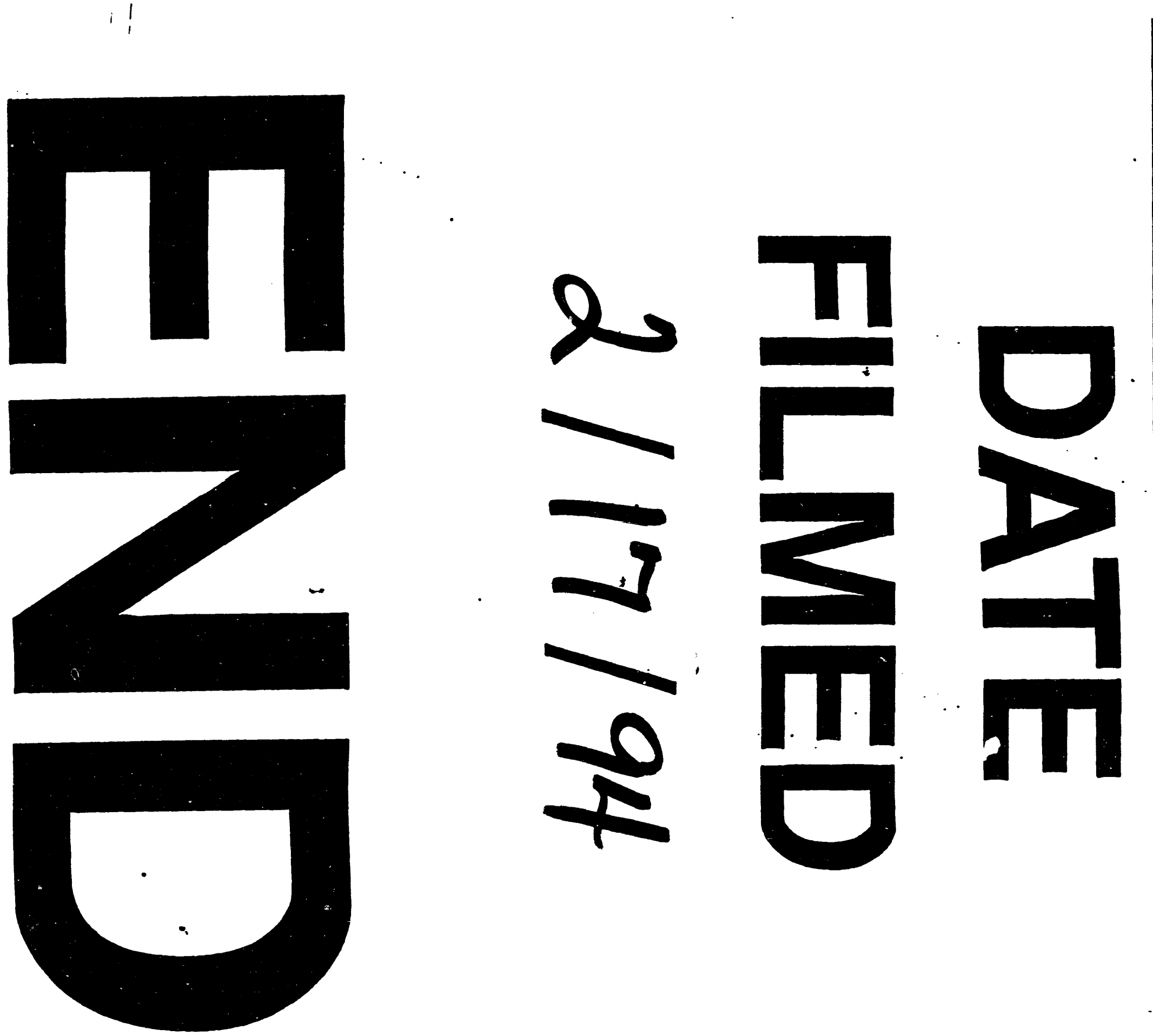

0

$+$

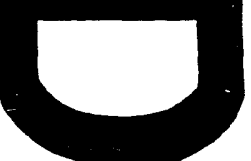


